\title{
Assessment of Behavioral Change in a Child Psychiatric Inpatient Program
}

\author{
Jane A. Waldron, Ph.D. \\ John A. Burns School of Medicine \\ Paula S. Nurius, M.S.W., M.A. \\ University of Michigan
}

\begin{abstract}
A behavioral rating scale, the Devereux Child Behavior Scale, was used to assess change in 55 children who were patients in a child psychiatric inpatient unit for an average of 3.9 months. The children were rated by unit staff on admission and at discharge, and by their parents prior to admission and at three and six months after discharge. The ratings indicate that the children did make statistically significant positive behavioral changes while in the hospital and that they continued to change up to six months post-discharge. Staff tended to rate the children as less disturbed than the parents but both groups of raters indicated significant change by their ratings.
\end{abstract}

Hospitalization of pre-adolescent children for psychiatric treatment is an intervention which arouses much feeling and debate in the mental health community. ${ }^{1,2}$ Who should be hospitalized, what kind of treatment should be provided and how long young children should be away from their homes, are all valid questions which have received a variety of answers depending on the informant. Whether inpatient treatment of psychiatrically disturbed children produces behavioral or dynamic change has received relatively little research attention to date. Although clinical observation tends to support the notion that positive change does occur during hospitalization there are only a few studies in the psychiatric literature which support the assumption. ${ }^{3,4,5}$

This research was supported in part by a grant from the Honolulu Chamber of Commerce, Public Health Fund. At the time this work was done, Dr. Waldron was the Director of the Leahi Hospital Children's Mental Health Unit, Honolulu, Hawaii. Address correspondence to Dr. Waldron at Kapiolani-Children's Medical Center, Child Guidance Center, 1319 Punahou Street, Honolulu, Hawaii 96826. At the time this work was done, Ms. Nurius was a Research Statistician at the State of Hawaii, Department of Health, Division of Mental Health, Honolulu, Hawaii. 
Research which focuses on the effectiveness of such treatment interventions is essential because of the risk of emotional trauma by the very separation of young children from their families and because of the high cost of inpatient psychiatric treatment.

Very real measurement problems plague attempts to clarify, define and examine the impact of psychotherapeutic strategies. A variety of methods have been employed to date in studying the effects of psychiatric hospitalization on patients. More studies have been done on adolescent populations than on child populations and in most cases combinations of interviews and behavioral scales or check lists have been used. $6,7,8,9,10$ The follow-up studies done on adolescent in-patient populations generally show favorable results with most studies demonstrating positive change in the majority of their ex-patients. One of the earliest outcome studies on latency aged children was done by Schaefer and Millman in $1973 .{ }^{4}$ In that study the investigators used a behavioral rating scale to assess changes in 35 boys ages 7 to 12. They found statistically significant improvement in behavior competence factors (good hygiene, control of bodily processes, regulation of the senses and development of basic social functioning) and less improvement in behavior control factors (internal control of impulses).

McConnell and Geddes in $1980^{3}$ evaluated an inpatient treatment unit for developmentally delayed children ages 2 to 15 using staff ratings of improvements in target behaviors. They followed their 102 child patients for three months after discharge and found that 71 percent were improved on at least one target symptom identified by the child's parents at admission. A study by Winsberg, et al, in $1980^{2}$ looked at differences in behavioral change between children treated in the community and children treated in a child psychiatric inpatient unit. The total sample included 49 children ages 5-13 who were treated for 6 months. Patients were randomly assigned to the two experimental groups. Measures for change included checklists, behavioral rating scales and semi-structured interviews. This study found significant change in the outpatient group. These researchers reported that the staff of the inpatient unit rated their patients as normal. Parental ratings for the same group of hospitalized patients were very different from the staff ratings. Thus, change for this group was hard to assess. The subjects did make significant improvement in some academic areas.

A five year outcome study by Schain, Gardella and Pon in $1982^{5}$ looked at 36 children in residential care at a children's treatment unit at Camarillo State Hospital. The mean age of subjects was 12.8 and 
the average length of stay in the program was 19.1 months. Data was gathered by structured interviews with parents or caretakers. This study found that most non-psychotic children were living in the community and adapting to community life five years after hospitalization. Violent behaviors were greatly reduced and the authors related the changes to the inpatient treatment.

\section{Setting}

The Children's Mental Health Unit is a twelve bed in-patient psychiatric treatment facility for emotionally disturbed children between the ages of four and twelve. At the time of the study, the Unit was a training site for a University School of Medicine, Department of Psychiatry, as well as for graduate students in Social Work, Psychology, Nursing, Special Education and Occupational Therapy. The Unit is located in a large State Hospital which houses patients in a variety of medical programs, i.e., tuberculosis, Hansen's Disease, extended care facility, intermediate care facility, etc. In 1976 a research team comprised of University faculty and Unit staff decided to explore the behavioral changes made by the child patients during hospitalization and after discharge. The purpose of the study was to learn about the impact of the hospitalization for the purpose of program evaluation.

\section{Methodology}

\section{Instrument}

In choosing an instrument for assessing change in the child patients, global rating scales, clinical assessments, projective tests and behavioral rating scales were considered. The team believed that a behavior rating scale offered the most reliable method of gathering data from several sources and that it would avoid the subjectivity of a more clinical assessment tool. The Devereux Child Behavior Scale (DCBS) was selected because it was developed in a residential treatment setting with child patients similar to the Unit's sample. ${ }^{11}$ The scale can be broken down into seventeen behavioral factors which include ten behavioral competency and seven control issues. Behavioral competence covers areas such as basic self-care, control of body processes, need for attention, need for independent mastery and basic social skills. Problems in behavioral competence, according to the scales' developers, Spivack and Spotts, are usually accompanied by intellectual deficiency or a deviation in the developmental process. Behavioral control factors are purported to tap 
various facets of the ability to regulate behavior, i.e., educational, emotional or social regulation problems. Children who have not evolved adequate ability to delay frustration or who have not developed an integrated system of inner controls are identified in the last seven factors.

Data on the scale were gathered by Spivack and Spotts from a study of 252 emotionally disturbed, atypical children ages 5 to 13 . DCBS ratings on a normal sample of 348 public school children between the ages of 5 and 12 were also gathered. It was felt that these samples could serve as comparison groups with the present study group in the data analysis. Reliability of rater, item and factor had been established for the DCBS. ${ }^{11}$ The Scale had also been used in two published studies, one by Schaefer and Millman which studied behavioral change in a residential treatment center for latency aged children and in another by Winsberg et $a{ }^{2}{ }^{2}$ in a study of home vs. hospital care for behavior disordered children. It was felt that comparison of the study results to previous study's result would enrich the findings and interpretation of the data.

\section{Design}

Data was collected from two sources; from the Unit staff and from the patients' parents. DCBSs were completed by Unit staff two weeks after admission and at the point of discharge. Rating scales were completed by parents prior to hospitalization and three and six month post discharge. Data was gathered on a total of 55 patients admitted to the Children's Unit from January 1977 to September 1978. Due to a variety of factors not all data was collected on each subject. Unit staff completed 47 DCBSs two weeks after admission and 50 at discharge. Parents completed 46 DCBSs prior to admission, 30 at three months and 20 at six months post-discharge. Some children changed homes or moved out of the area before follow-up data could be obtained.

\section{Results}

\section{Description of Sample}

The 55 children in the study ranged in age from 4 years 11 months to 13 years 1 month at the time of admission to the Unit. The median age was 9 years and the mean age was 8 years 11 months. 20 percent of the children were female and 80 percent were male. Table 1 indicates ethnicity of the sample and Table 2 describes the referral sources of the subjects. 
Table 1

Ethnicity
Table 2

Referral Source

Caucasian

Part Hawaiian Mix

$56 \%$

$20 \%$

Non-Hawaiian Mix

Japanese

Black

Chinese

Portuguese

Korean
$11 \%$

$4 \%$

$4 \%$

$2 \%$

$2 \%$

$2 \%$
State Mental Health System

Private psychiatrist

Military psychiatrist

Parents

Child protective services

Private social agencies

Welfare Department

Private social workers

Private physicians

Department of Education
$22 \%$

$15 \%$

$11 \%$

$11 \%$

$11 \%$

$6 \%$

$5 \%$

$4 \%$

$2 \%$

$2 \%$

The reasons for hospitalization as assessed by the research team were most often related to severe behavioral disturbance. See Table 3.

\section{Table 3}

\section{Reasons for Hospitalization*}

Aggressive Behavior

Peer conflict

Severe rage/Temper tantrums

Oppositional behavior

Delinquent behavior

Run away behavior

\section{$55 \%$}

$42 \%$

$36 \%$

$24 \%$

$7 \%$

Withdrawn or Depressed Bbehavior

Other Problems

Self destructive behavior

$11 \%$
$9 \%$
$5 \%$
$4 \%$
$2 \%$

School Problem Academic/Behavior $22 \%$ Distractibility $22 \%$ School refusal $2 \%$

Extreme dependence

Depression Withdrawal

Suicidal threat/attitude

Parents need relief

$24 \%$

Enuresis/Encopresis

$20 \%$

Potential for child abuse $\quad 16 \%$

Severe lying

$13 \%$

Unusual language

$13 \%$

Sexual behavior

Preparation for placement $5 \%$

Seizures $\quad 2 \%$

Diagnostic evaluation

$2 \%$

* Since children were often referred for more than one problem, the percentages shown do not total $100 \%$. 
The diagnostic classification used was that proposed by the Group for the Advancement of Psychiatry. ${ }^{12}$ Table 4 presents the percentage of subjects according to diagnosis.

Table 4

Diagnosis

$\begin{array}{lr}\text { Reactive disorder } & 2 \% \\ \text { Developmental deviation } & 36 \% \\ \text { Neurotic disorder } & 11 \% \\ \text { Personality disorder } & 45 \% \\ \text { Psychophysiologic } & 2 \% \\ \text { Psychosis } & 2 \% \\ \text { Mental retardation } & 2 \%\end{array}$

The diagnosis used for research purposes was that given a child two weeks after entry into the hospital. The distribution of diagnoses, as would be expected, was somewhat associated with the age of the child. Personality disorder was used most often with children over nine years of age and developmental deviation tended to be used with younger children. The most common developmental deviation diagnosis was in the social area and the most common personality diagnosis was tension discharge disorder.

The length of time children in the sample were hospitalized ranged from two weeks to ten months. Thirty six percent of the sample were in the hospital less than three months and 64 percent were hospitalized for three months or more. The average length of stay for all children in the sample was 3.9 months.

\section{Data Analysis}

Means and standard deviations for each of the DCBS 17 factors were calculated for each of the rater groups, Unit staff and patients' parents at each of the data collection points. Staff data was collected two weeks after admission and again at discharge. Parents data was collected prior to admission and at three and six months post discharge. Change scores (post-score minus pre-score) were calculated and analyzed using a one-tailed Student's t test for correlated means comparisons at the .05 level of significance. Parental and staff means were compared with similar data obtained from an atypical and normal sample by the DCBS's developers, Spivack and Spotts. Comparisons were then made with the two other studies ${ }^{4,2}$ which used the same instrument, DCBS, in collecting data from somewhat similar samples. 


\section{Children's Unit Results}

In the Unit sample, parents and staff means were significantly different from each other, with parents rating their children as more disturbed than the Unit staff rated them. Both groups of ratings, however, indicated that the subjects behavior had improved. Staff ratings made during hospitalization indicated statistically significant positive change for eight DCBS factors. Parental ratings show five factors significantly changed at three months following discharge and nine factors reaching significance at six months following discharge. Both parents and unit staff rated significant change in five factors at the six month follow-up period. Table 5 indicates the factors for each group of raters which had significantly changed scores.

Table 5

DCBS Factors Rated as Significantly Changed

Factors rated by parents* and staff ${ }^{+}$as improved

Distractibility

Pathological use of senses

Emotional detachment

Inability to delay

Social aggression

Factors rated by parents and staff as not improved

Poor self care

Messy/Sloppy

Inadequate need for independence

Need for adult contact

Impulse Ideation
Factors rated by parents as improved*

Poor coordination

Incontinence

Proneness to emotional upset

Anxious-fearful ideation

Factors rated by staff as improved $\dagger$

Social isolation

Unresponsive to stimulation

Unethical behavior

*At 6 months after discharge from hospital.

$\dagger$ At discharge from hospital.

\section{Comparison of Unit and Devereux Data}

The atypical sample gathered by Spivack and Spotts was called DOWB, a name derived from the names of the four treatment centers from which the data were drawn, Devereux School, Oakburne Hospital, Woods School and Emma Pendleton Bradley Hospital. The DOWB sample was roughly comparable to the Unit sample with 
regard to age but was clearly a more psychiatrically disturbed population. Diagnoses for the DOWB sample were distributed as follows: personality disorder, 29 percent; CBS with behavior reactions, 8 percent; CBS without behavior reactions, 22 percent; schizophrenic reactions, 22 percent; Other, 22 percent. The DOWB ratings were made by the various treatment center staffs at varying points during hospitalization.

A comparison of the Unit sample with the atypical DOWB (252 subjects) and the Normal (348 subjects) samples reported by Spivack and Spotts was made using the sum of the means for each group. Although the parental means on the DCBS at admission $(\Sigma M=250.1)$ and the staff means at two weeks after admission ( $\Sigma M=176.6)$ were significantly different from each other, neither was significantly different from the DOWB means $(\mathrm{M}=212.1)$. The parental ratings of the Unit sample at the point of admission indicate more severely disturbed behavior than the ratings of the DOWB sample but the difference was not statistically significant. On 15 out of 17 factors, parents rated their children as more disturbed than the DOWB staff rated their subjects. Unit staff rated their patients as less disturbed than the DOWB sample on 15 of 17 factors.

Parental ratings of the Unit sample at the three month follow-up point $(\Sigma M=220.3)$ reflect perceptions of less disturbance than at admission and ratings by parents at six month follow-up point $(\Sigma \mathrm{M}=$ 207.3) are slightly below the DOWB ratings. None of the parental ratings at any point come very close to the ratings made on the Normal sample $(\Sigma M=149.8)$. Parental ratings of the Unit sample at three months indicate a perception of greater behavioral disturbance than the ratings of Unit staff at discharge $(\Sigma M=168.0)$ but the difference is not statistically different from ratings of the Normal group whereas the parental three month post-discharge ratings are significantly higher than those of the Normal sample.

In summary, when compared with Unit staff ratings, the parental ratings were much higher, indicating that parents in the study saw their children as more behaviorally disturbed than the staff who treated them. In addition, parents ratings were higher than staff ratings in the DOWB treatment centers. Unit staff ratings at the point of discharge are close to the ratings for the Normal sample and are significantly different from the parental ratings. 


\section{Discussion}

The findings of the current study must be considered against the philosophy and objectives of the Children's Mental Health Unit's treatment program. Most of the children referred to the Unit were children whose behavior was impulse ridden, antisocial and negativistic. These children were disturbing to their families, schools and communities. As a result the program was set up to provide clear and strong structure for the child patients and to offer close supervision at all times. The child's day was a full one with few choices permitted. Support and guidance were provided liberally by the child care staff and individual, group and family therapy were a part of the treatment regime. In short the Unit milieu was designed to help impulsive children succeed. Out-patient treatment following dicharge was either provided by the Unit staff or referrals were made to community resources.

Given the amount of structure in the program and the high degree of supervision and support, it is not surprising that the DCBS ratings for distractibility, inability to delay frustration, social aggression and unethical behavior showed statistically significant change scores. These factors tended to be those in which change from extreme deviance towards normal was the greatest. Parents ratings also indicated significant change in distractibility, inability to delay frustration and social aggression.

Pathological use of the senses, emotional detachment, social isolation and unresponsive to stimulation were the factors which Unit staff rated as significantly improved. This finding is not surprising in view of the high demand and support for social participation offered to children in a group treatment program. As noted earlier, a good part of each child's day is spent with the group in highly-structured, wellsupervised activities which were designed to reduce conflict, provide successful group experiences and to teach manual, academic and social skills. Parental ratings indicated significant change scores in only two of these areas, pathological use of senses and emotional detachment.

Poor self care, poor coordination, incontinence and messiness/sloppiness are factors which did not show statistically significant change when rated by Unit staff. These factors are related to behaviors which were not problems for the majority of patients in the study sample and the program doesn't tend to stress these areas. It is clear, however, that there is a small group of patients for whom these issues are a problem and that improvement in these behaviors for some children 
needs to receive program focus. Parents rated significant improvement in poor coordination and incontinence at six months after discharge.

Factors which did not show statistically significant change scores when rated by Unit staff were anxious-fearful ideation, impulse ideation and proneness to emotional upset. This finding indicates that although acting-out behaviors tended to decrease, internal and verbal representations of the underlying insecurities, fears and conflicts remained. It may be that the duration of in-patient treatment is too short a time to expect change in these internal emotional areas. Two of the three factors lanxious-fearful ideation and proneness to emotional upset) were rated as significantly improved by parents at six months after discharge. The third factor, impulse ideation showed a trend towards improvement according to the parental ratings.

The remaining two factors which did not show statistically significant change scores when rated by Unit staff and by parents were inadequate need for independence and need for adult contact. These two factors both relate to the lack of autonomous development. The lack of resolution of such problems may be associated with the Unit's program objectives which stress control issues more than autonomy issues. Some adaptation within the hospital program is possible to accommodate children as they improve their ability to control themselves and to support those who need a more flexible environment, but this is still within the context of a highly structured and closely supervised setting. This finding also needs to be taken into account in program planning.

The finding that parents rated their children as significantly more disturbed than did the Unit staff is interesting. It is certainly plausible that parents would express a more negative view of the extent of their children's problems than would Unit staff since the parents had had to live and contend with the difficulties on a daily basis, often with little support or guidance. Inpatient psychiatric hospitalization represents a 'last resort' measure by the majority of parents who are desperate for a solution and frustrated by their previous attempts at mastering their parent-child problems. The study by Winsberg et $\mathrm{al}^{2}$ also reported differences in parental and staff ratings on the same children with staff rating children as less disturbed. That group concluded that staff may have a greater tolerance for behavioral deviance or that the different environment might well stimulate different behaviors. Attempts to compare staff and parental ratings is clearly problematic. The question may not be so much who is most accurate but rather are the 
two groups rating somewhat different behaviors using different standards. It is also highly possible that the child behaves differently in different settings.

The data indicated that the subjects continued to improve steadily after discharge and this corresponds to findings of studies of adolescent inpatient treatment facilities. ${ }^{6} 15$ out of 17 factors changed in a positive direction as assessed by parents from the three month to the six month follow-up point and nine of these did so to a statistically significant degree. Again, as with staff ratings, parental ratings indicated significant changes in factors relating to behavioral competence (distractibility, pathological use of senses, emotional detachment, poor coordination, incontinence) and behavioral control (proneness to emotional upset, anxious-fearful ideation, inability to delay frustration, social aggression). This represents a different finding from the Children's Village study where the most improvement was in behavioral change factors. ${ }^{4}$

\section{Conclusions}

Analysis of the DCBS ratings by Unit staff and parents supports the notion that children in this sample did make significant changes during and following discharge from the hospital and that many of those changes were maintained or increased throughout the six month post-discharge period. Areas of strength for this in-patient treatment program appear to be in assisting patients achieve emotional connectedness and control of impulsivity and aggressiveness. There seems to be relative weakness in the program's effectiveness in assisting patients in developing some self care skills and in achieving emotional and behavioral autonomy. The implications of these findings for program planning are important since without some formal way of assessing a program's effectiveness there is a tendency for inertia and complacency to interfere with a program's ability to keep pace with and adapt to its patients' needs.

Different treatment programs will have different strengths and weaknesses depending on staff and program strengths. This makes it difficult to speculate on the overall strengths of inpatient psychiatric treatment. Despite the measurement problems however more studies of behavior change in treatment settings dealing with children are needed and these studies need to include control groups of similar patient groups untreated or treated in outpatient settings in order to 
add to our knowledge about the appropriateness and specificity of inpatient treatment.

The type of approach being discussed requires a clear commitment to research by clinical settings and such commitments in time, energy and money are especially difficult to secure in these days of reduced spending for mental health research. Without continued research, however, it will not be possible to use inpatient treatment as an option in the treatment of severely disturbed children.

\section{References}

1. Moultrie MB and Carek DJ: Short term inpatient treatment of children and adolescents. The Journal of the South Carolina Medical Association 79(1):30-3, 1983.

2. Winsberg BG, Bialer I, Kupietz S, et al: Home vs hospital care of children with behavior disorders. Archives of General Psychiatry 37(4):413-8, 1980.

3. McConnell MM and Geddes G: Evaluation of an assessment and training unit for developmentally delayed children. New Zealand Medical Journal 92(666):161-4, 1980.

4. Schaefer CE and Millman HL: The use of behavior ratings in assessing the effect of residential treatment with latency age boys. Child Psychiatry and Human Development 3(3):157-64, 1973.

5. Schain RJ, Gardella D and Pon J: Five-year outcome of children admitted to a state mental hospital. Hospital \& Community Psychiatry, 33(10):874-8, 1982.

6. Fineberg BL, Sowards SK and Kettlewell PW: Adolescent inpatient treatment: a literature review. Adolescence 15(60):913-25, 1980.

7. Gossett JT, Barnhart FD, Lewis JM, et al: Follow-up of adolescents treated in a psychiatric hospital: measurement of outcome. Southern Medical Journal $73(4): 459-66,1980$.

8. Guy ME and Moore S: The goal attainment scale for psychiatric inpatients. Quality Review Bulletin 8(6):19-29, 1982.

9. Machalow R: Review of the literature: follow-up psychiatrically hospitalized adolescents. Adolescence 17(68):789-99, 1982.

10. Welner A, Welner Z and Fishman R: Psychiatric adolescent inpatients. Archives of General Psychiatry 36(6):689-700, 1979.

11. Spivack G and Spotts J: Devereux Child Behavior Rating Scale Manual, Devon, Pa., Devereux Foundation, 1966.

12. Group for the Advancement of Psychiatry: Psychopathological Disorders in Childhood: Theoretical Considerations and A Proposed Classification, New York, Mental Health Materials Center, Inc., 1966. 